\title{
Engine System Model Development for Nuclear Thermal Propulsion
}

\author{
Karl W. Nelson, Ph.D. ${ }^{*}$ and Steven P. Simpson, M.S. ${ }^{\dagger}$ \\ NASA - Marshall Space Flight Center, Huntsville, AL, 35812
}

\begin{abstract}
In order to design, analyze, and evaluate conceptual Nuclear Thermal Propulsion (NTP) engine systems, an improved NTP design and analysis tool has been developed. The NTP tool utilizes the ROCket Engine Transient Simulation (ROCETS) system tool and many of the routines from the Enabler reactor model found in Nuclear Engine System Simulation (NESS). Improved non-nuclear component models and an external shield model were added to the tool. With the addition of a nearly complete system reliability model, the tool will provide performance, sizing, and reliability data for NERVA-Derived NTP engine systems. A new detailed reactor model is also being developed and will replace Enabler. The new model will allow more flexibility in reactor geometry and include detailed thermal hydraulics and neutronics models. A description of the reactor, component, and reliability models is provided. Another key feature of the modeling process is the use of comprehensive spreadsheets for each engine case. The spreadsheets include individual worksheets for each subsystem with data, plots, and scaled figures, making the output very useful to each engineering discipline. Sample performance and sizing results with the Enabler reactor model are provided including sensitivities. Before selecting an engine design, all figures of merit must be considered including the overall impacts on the vehicle and mission. Evaluations based on key figures of merit of these results and results with the new reactor model will be performed. The impacts of clustering and external shielding will also be addressed. Over time, the reactor model will be upgraded to design and analyze other NTP concepts with CERMET and carbide fuel cores.
\end{abstract}

\section{Nomenclature}

$\begin{array}{ll}E R & =\text { nozzle expansion ratio } \\ I M L E O & =\text { Initial Mass in Low Earth Orbit } \\ I s p & =\text { specific impulse } \\ k_{e f f} & =\text { neutron production rate / neutron loss rate } \\ L / D & =\text { length to diameter ratio } \\ P c & =\text { chamber pressure } \\ T / W & =\text { thrust to weight ratio } \\ \Delta P & =\text { change in pressure (exit }- \text { inlet) }\end{array}$

\section{Introduction}

TN 2004, NASA announced its new Vision for Space Exploration. With this renewed interest in manned missions 1 to Mars, Marshall Space Flight Center (MSFC) has initiated several tasks that support nuclear thermal propulsion (NTP) development. The primary objective of the engine system modeling, analysis, and design task is to develop the process and tools necessary to design, analyze, and evaluate conceptual NTP engine systems. Secondary objectives of this task were to perform trades, define requirements, and identify technology gaps and risks, all at both the system and component levels. Initially, engines with graphite or composite prismatic fuel reactor cores, similar to those developed during the Rover and Nuclear Engine for Rocket Vehicle Application (NERVA) programs, would be investigated. Later, engine concepts with potentially higher performance, such as those with

\footnotetext{
* Engine Systems Engineer, Nuclear Systems Branch, Mail Code: ER24.

${ }^{\dagger}$ Systems Analysis Engineer, Nuclear Systems Branch, Mail Code: ER24.
} 
CERMET and carbide fuel cores, would be investigated. In order to accomplish these goals, an improved NTP engine system design and analysis tool was needed.

From a mission and vehicle perspective, the primary engine performance requirements are thrust, weight (or thrust to weight ratio), specific impulse, and envelope. Other top-level requirements that need to be considered in the engine design are: number of engine burns during the mission; duration of each burn; engine allocated reliability and cost; safety, and risks. The definition of component level requirements is also of significant importance and was identified as an objective of the engine system task. Therefore, it was desired that the NTP engine system tool provide a complete power balance and contain integrated non-nuclear component and reactor models, along with system reliability and cost models. Since Mars mission and vehicle system studies conducted over the years have resulted in recommendations of a wide range of engine thrust levels, the system tool needed to be very flexible in terms of sizing. Another desirable feature of the tool was the ability for component and systems engineers to easily retrieve and evaluate the results for their area of expertise in order to make recommendations. The tool also needed to run quickly on a typical desktop computer in order to perform design trades and be useful to others. Finally, it was preferred that the tool have the capability to perform transient analyses for more detailed design at a later date.

\section{System and Reactor Model Selection}

\section{A. System Model Selection}

The first step in generating the NTP engine system tool was to select a system level modeling tool and a reactor model from existing codes.

A key element of the approach for the NTP engine system task was to leverage a proven trade study technique used for chemical rocket engines. This process begins with a number of potential engine concepts. The process then involves assessing the concepts based on figures of merit (FOMs), downselecting a portion of the concepts, adding more detail to those concepts, and repeating until a desired subset of concepts remains or a predetermined level of fidelity is achieved. The initial loops through this process would utilize an engine system level design tool with integrated component models.

A recent upperstage chemical rocket engine study performed at MSFC followed this process. The study utilized a newly developed Microsoft Excel based engine system design tool called Propulsion Sizing, Thermal, Accountability, and weight Relationship (P-STAR). ${ }^{1}$ P-STAR addressed the integration of system performance, reliability, and cost models into a single tool, and had an interface amenable to a multi-disciplined design team. P-STAR was the proposed system tool for the NTP engine system task. However, after additional consideration, it was determined that the required modifications to P-STAR in order to model NTP systems and the learning curve to use P-STAR would not be an efficient use of time. In addition, P-STAR does not currently have the capability to perform transient analyses. Thus, it was decided that the initial step should be a more conventional modeling approach. Nonetheless, this previous study showed that the Excel interface was a very beneficial feature for a design and analysis tool.

Nuclear Engine System Simulation (NESS) ${ }^{2}$ is a system level NTP code developed by Science Applications International Corporation (SAIC) for Glenn Research Center (GRC). It includes a reactor model called Enabler, which was developed by Westinghouse Electric Corporation and is based on the Rover/NERVA reactor designs. NESS was considered for the system tool for the NTP engine system task, but was not selected because it is not a modular code, and it is only set up for a limited number of cycles and flow paths. Integrating alternative component models or additional cycles into NESS would be challenging and time consuming. However, the Enabler reactor model within NESS appeared to be quite useful and at a reasonable design level.

ROCket Engine Transient Simulation (ROCETS) ${ }^{3}$ is the primary system level code used at MSFC. It has been used successfully at MSFC for rocket engine development since 1992 on the Space Shuttle Main Engine (SSME), RL-10, MC-1, Integrated Powerhead Demonstrator (IPD), and many other projects. ROCETS is a FORTRAN based modular code with text input and a Newton/Raphson solver. As the name implies, it is also capable of performing transient analyses. Although ROCETS had many of the desired features, it required the integration of a reactor model and it lacked a friendly interface for a multi-disciplined team.

The decision was made to select ROCETS as the system level tool and integrate the Enabler reactor modeling approach from NESS. Improved non-nuclear component models would also be added to ROCETS. Ideally, the communication between the system code, non-nuclear component codes, and reactor code would be highly coupled, as shown in Fig. 1, to facilitate a flexible and physically accurate system model. The output from ROCETS would be imported into a separate Excel spreadsheet for each engine case modeled. Within the spreadsheet, the results for each subsystem would be contained in individual worksheets. In addition, sketches and plots could also be included in the worksheets to represent data graphically as an aid to the engineers. Once a spreadsheet template was created, 
spreadsheets could be produced for each case by simply pasting the ROCETS output for that case into a specific location on one of the worksheets. The combination of ROCETS and Excel allows the option of integrating other models, such as reliability and cost, either into ROCETS or into the spreadsheet, depending on the model source and user preference.

\section{B. Enabler Reactor Model}

The Enabler reactor model was used as a guide to generate the current nuclear reactor model implemented in ROCETS. The Enabler code has many underlying assumptions and nuances that must be thoroughly understood before any results can be interpreted.

Unfortunately, as will be discussed and is being remedied, many of the computations in Enabler are based on fixed empirical data rather than physics

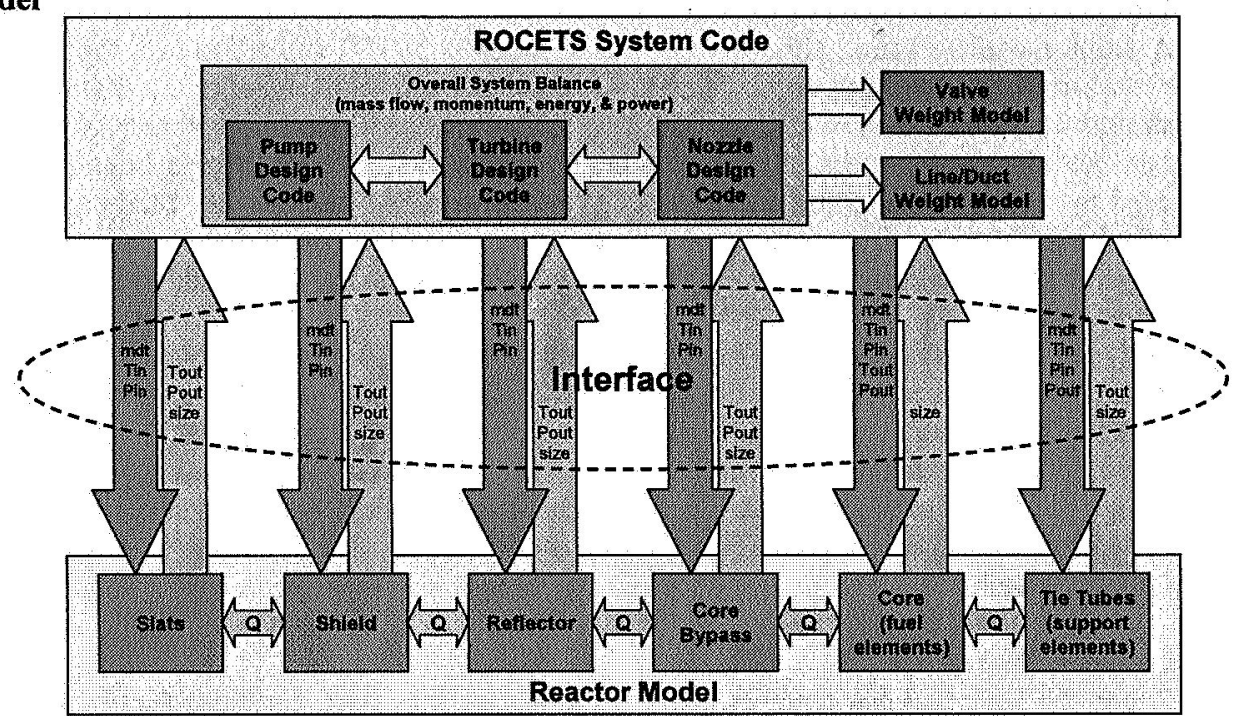

Figure 1. Communication Between Models (Ideal). based models of the reactor components and inter-component communications shown in Fig. 1.

Enabler essentially models a reactor based on that of the Phoebus-2A engine, which was designed and built during the Rover/NERVA program. This reactor core design includes fuel elements with a hexagonal (prismatic) cross-section and several hydrogen coolant passages. The fuel elements are supported by tie tubes, which also contain moderating material to help thermalize the reactor. Subsequent NERVA reactor designs utilized this same approach. ${ }^{4}$ Enabler allows specific fuel element to tie tube ratios of 2:1, 3:1, or 6:1. The tie tubes are cooled with hydrogen in a down and up pass configuration. The tie tube flow, which can be used to power the turbomachinery, is eventually passed through the core. Enabler uses a fixed power per fuel element, and the fuel elements have a given length, given flat to flat dimension, and a 19 coolant-hole arrangement through their entire length. Based on the power requirement, which is a function of the thrust, chamber temperature, chamber pressure, and expansion ratio of the nozzle, Enabler will size the core of the reactor. Based on this size, other associated hardware within the reactor is sized: reflector, control drums, pressure vessel, support structures, support plates, flow baffles, instrumentation rings, and several other pieces of hardware. The nuclear reactor model implemented in ROCETS essentially duplicates this sizing routine.

Furthermore, Enabler has a thermal hydraulic model of the coolant holes in the fuel elements. The thermal hydraulic model assumes a generic cosine axial power distribution curve with a peaking factor of 1.2. The heat transfer coefficient is calculated from McCarthy-Wolf..$^{5}$ The thermal hydraulic model also assumes an empirical relationship for the fuel element center line temperature. Pressure drop is calculated using friction factor formula from Haaland. ${ }^{6}$ The nuclear reactor model implemented in ROCETS duplicates this model.

Unlike the core thermal hydraulic model, the tie tube model in Enabler assumes a pressure drop and heat input into the tie tube flow. The nuclear reactor model implemented in ROCETS uses similar techniques as in the fuel element model to determine a pressure drop and a temperature of the fluid in the tie tube.

Enabler also models an internal shield that provides a limited amount of protection from radiation. The shield has two components: borated aluminum titanium hydride (BATH) and lead. The thickness of each component is based on a given power level, fluence level, and dose plane distance from the center of the core. The diameter of the shield is equal to the reflector outer diameter. The nuclear reactor model implemented in ROCETS duplicates this function.

One more important feature of Enabler is the criticality criteria. This criterion, which states whether or not the reactor is critical, is based on the element ratio and fuel volume. The basis for the criteria and where it should be 
applied is not fully understood. Nevertheless, the nuclear reactor model implemented in ROCETS employs the same criteria.

\section{Non-nuclear Component Models}

\section{A. Pump and Turbine Models}

A pump meanline sizing code developed internally at MSFC is used to size the pumping elements and predict their performance. Minimum inputs of inlet conditions, required headrise, mass flow rate, shaft speed, and constraints on suction parameters, specific speed, tip speed, and several geometry ratios are used to start the sizing process. Standard pump meanline calculations in conjunction with specific turbopump design rules and constraints are used to set the required number of stages, and the major diameters and blade angles of the pump inducer and impeller(s). Suction performance capability (cavitation) is estimated from empirical curves, inlet and discharge velocity triangles are computed for each element, efficiencies are predicted, and estimates of turbopump size and weight are made.

Turbine modeling is done with a code adapted by MSFC from TURBAN, a code developed by GRC. TURBAN computations are based on mean diameter flow properties and stage average velocity diagrams. Details of the TURBAN's computation methods, inputs and outputs can be found in Refs. 7 and 8. TURBAN is called as a subroutine and exit radius ratio is varied to determine the maximum efficiency for a given set of input conditions. The program is used to calculate exit annulus dimensions, efficiencies, and exit pressure and temperature. The loss coefficient, KLOSS, is set at 0.55 based on modeling of several recent turbines designed for rocket engine turbopump assemblies.

\section{B. Chamber/Nozzle Model}

Due to the high chamber temperatures $(\sim 2700 \mathrm{~K})$ and long burn durations for the engine $(\sim 1 \mathrm{hr})$, the nozzle must be cooled to maintain its integrity. The normal procedure for modeling cooled nozzles at MSFC for preliminary design is to approximate the heat load and pressure drop based on previously built cooled nozzles. After the preliminary design phase, Rocket Thermal Evaluation (RTE) ${ }^{9}$ is used to perform a detailed design of the cooled nozzle. RTE is a computationally intensive program that generates a detailed mesh of the nozzle coolant geometry. The chamber/nozzle design code implemented in ROCETS attempts to do a fairly detailed design of the cooled nozzle without having the penalty of running a detailed code such as RTE.

The nozzle design code employs a fin analysis to determine the heat transfer out of the chamber/nozzle. Fig. 2 shows this model pictorially. In the analysis, the channels are discretized lengthwise. Therefore, an exact, implicit equation for the heat transfer and temperature distribution for the cooling structure is obtained. This analysis provides a fast and easy method to determine the heat transfer solution to the chamber/nozzle.

The hot gas heat transfer shown in Fig. 2 is calculated using the $\mathrm{Bartz}^{10}$ relationship for a rocket nozzle and chamber. The heat transfer into the coolant is calculated using the Dittus-Boelter ${ }^{11}$ correlation with entrance and

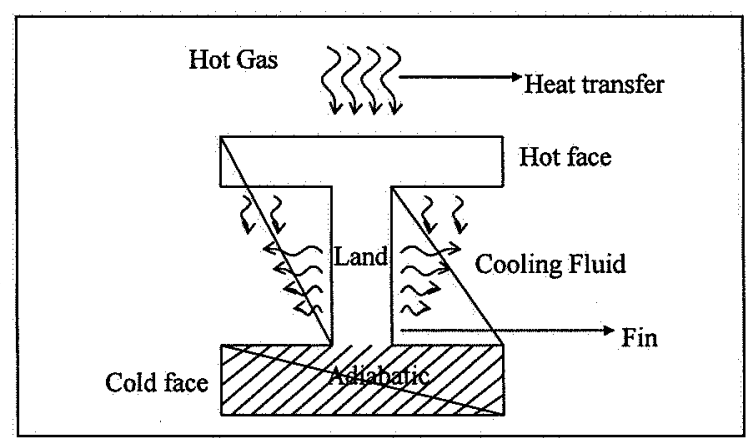

Figure 2. Chamber/Nozzle Fin Analysis Diagram. curvature effects added. The pressure drop in the coolant channels is calculated by a relationship developed by Chen ${ }^{12}$ with entrance and curvature effects added.

In order to capture the correct geometry of a real nozzle, the geometry is calculated using the Rao ${ }^{13}$ correlations for nozzle contours. The cold face thickness is determined by stress limits of the materials. Since the exact sizes of the chamber and nozzle are known, a precise weight can also be determined.

\section{Weight Models for Valves, Lines, and Ducts}

The valve weight model and line and duct weight model are based on historical data from the SSME, RS-68, MC-1, and RS-84 engines. For the NTP tool, only the hydrogen system valves in the database are utilized. Similarly, the line and duct weight is based on the total line and duct weight of only the hydrogen systems in the SSME and RS-68. The individual valve weight and the total line and duct weight are scaled based on flow rate. 


\section{Hydrogen Properties}

The hydrogen properties model was taken from the NESS tool. The model provides properties that match the National Bureau of Standards (NBS) data across the pressure range of 29 to 2320 psia $(0.2$ to $16 \mathrm{MPa})$ and the temperature range of 24.8 to $5400{ }^{\circ} \mathrm{R}(13.8$ to $3,000 \mathrm{~K})$. Above this pressure and temperature range, data generated from One Dimensional Equilibrium (ODE) is used for hydrogen properties.

\section{Additional Models}

\section{A. System Reliability Model}

The reliability modeling effort consists of defining mission failures, developing a failure logic model for an assumed mission, quantifying the failure events in the model, and executing the model to derive results in support of design trades. A conceptual-level working failure modes and effects analysis (FMEA) will be developed for the reactor subsystem and for all other NTP engine subsystems and components. The FMEA will serve (1) to identify the failure modes or events to be included in the failure logic model and (2) as the initial conceptual baseline FMEA for further development as desired or required.

A failure logic model will be developed based primarily on the conceptual architecture, its critical failure modes as determined by the FMEA, and the mission timeline as adapted from NTP Mars mission studies. The failure logic model will order NTP failure modes or events along the mission timeline by engine phases repeated per burn sequence (e.g., dormancy, checkout, startup, mainstage, shutdown, cooldown). Probability models will be developed for the failure modes and events in the failure logic model. These models will be based on SSME failure data for all components excluding the reactor subsystem. The SSME data will be scaled to the NTP components using configuration and performance deltas between the NTP engine concept and SSME. The output of the individual component and subsystem failure models will be aggregated through the failure logic to provide an engine-level reliability and risk estimate.

All modeling will be analytical and/or simulation developed in Microsoft Excel. The reliability model will be linked to the ROCETS system tool through a set of defined output variables from ROCETS. The model should allow for future expansion capability for additional design detail or to capture mission event complexity.

\section{B. External Shield Model}

The external shield model XTSHIELD ${ }^{14}$ calculates the thickness, geometry, and mass for external gamma and neutron shielding of a space based nuclear reactor. The shielding routine provides for three shielding configurations, shadow shield, $2 \pi$ shield for propulsion, and $2 \pi$ shield for surface power. The routine also allows for multi-engine configurations. The shadow shield for a multi-engine configuration includes shielding between the engines to ensure no unshielded, forward scattering between the engines. The shape of the shadow shield covering the two engine cluster is rectangular with a hemisphere on each end. For the three engine cluster, the shadow shield is triangular with a $120^{\circ}$ arc at each corner. For a multi-engine configuration with $2 \pi$ shields, each engine has an identical $2 \pi$ shield.

The shadow shield option allows for radiation attenuation in only one direction and within a cone half-angle specified as an input. The $2 \pi$ shield extends shielding down the length of the reactor, leaving only one end unshielded. The surface power configuration attenuates radiation forward and to the sides equally. The propulsion configuration attenuates radiation to the side two orders of magnitude less than the attenuation forward, towards equipment and crew. This reduction in shield thickness reduces mass, but still provides sufficient side shielding for neutronic decoupling between the reactors and sufficient attenuation of radiation forward caused by scattering.

The thickness of the neutron and gamma shield for a given power level, payload distance, and dose limit is determined based on the neutron and gamma attenuation coefficients for Lithium-Hydride and Tungsten, the only materials currently in the model. This technique is drawn heavily from the ALKASYS ${ }^{15}$ code, originally developed at Oak Ridge National Laboratory (ORNL) and enhanced by MSFC. The thickness calculations assume the gamma shield is immediately forward of the reactor pressure vessel, followed immediately by the neutron shield. The remaining shield geometry and mass is then calculated from the shield thicknesses, the cone half angle, and the reactor geometry. 


\section{Model Verification}

\section{A. Reactor Model Verification}

A comparison was made between the output of the Enabler reactor model used in NESS and the reactor model implemented in ROCETS. The ground rules of the reactor model verification were to (1) use the closed expander cycle with the turbine supply from the tie tubes, (2) compare cases from 50 to $250 \mathrm{klbf}$ thrust with a scaling factor of 1, (3) compare only the reactor parameters, and (4) keep all of the reactor inputs the same.

Most of the comparisons shown in Figs. 3a-f were very favorable. The power and number of elements (Figs. 3a, c, and d) diverged some at the upper thrust range. This error is approximately $2 \%$ and is primarily due to the different methods for calculating chamber pressure $(\mathrm{Pc})$. The reactor model implemented in ROCETS uses the ODE code for chamber conditions, whereas Enabler uses a curve fit. In addition, the reactor model implemented in ROCETS uses a smaller amount of core bypass flow. The difference in pressure drop through the reactor for the two codes (Fig. 3f) is within $10 \%$.
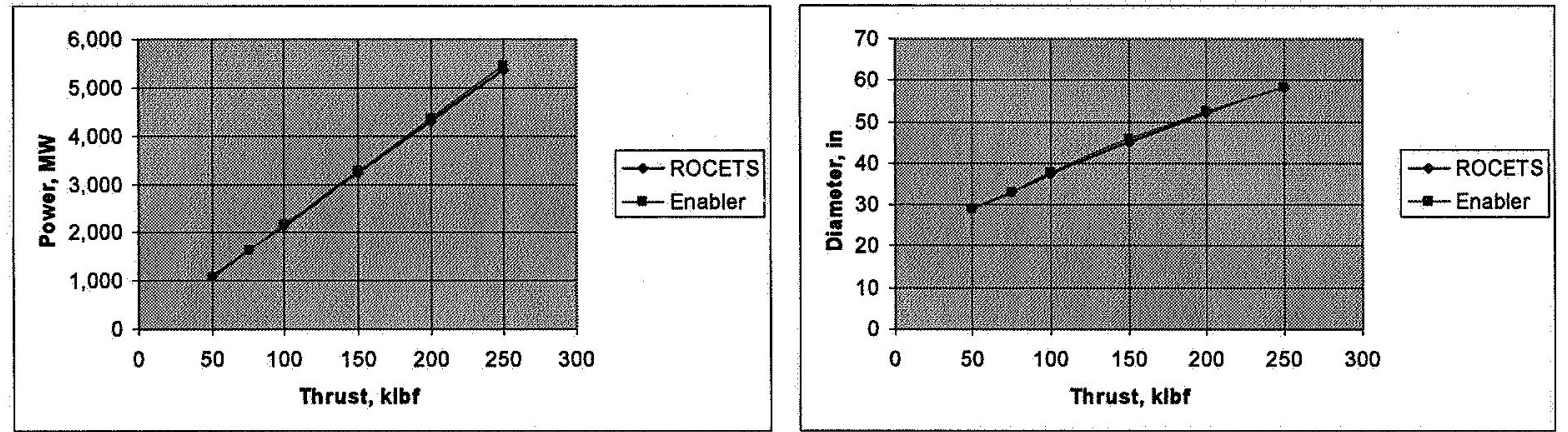

a)

b)
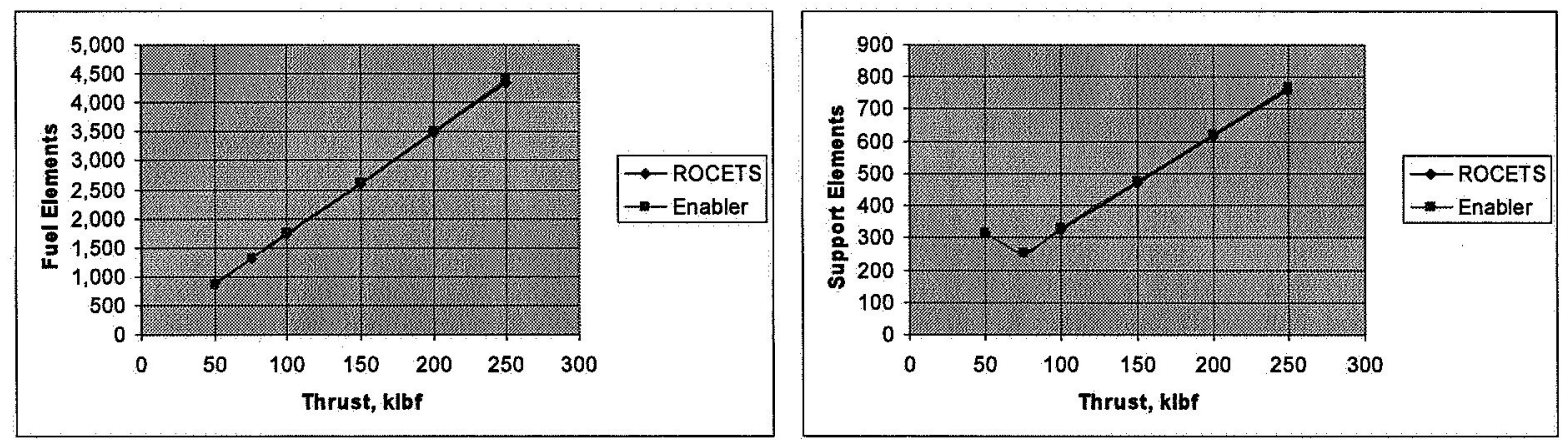

c)

d)
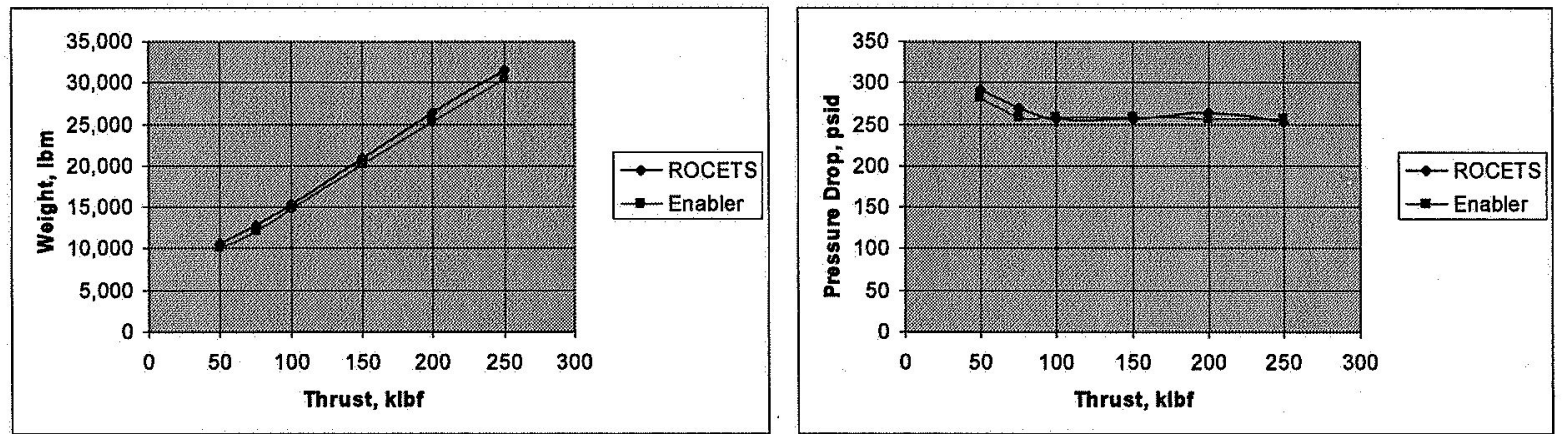

e)

f)

Figure 3. Comparison of Reactor Model in ROCETS to Enabler. 


\section{B. Chamber/Nozzle Model Verification}

In order to determine the accuracy of the chamber/nozzle cooling model implemented in ROCETS, a comparison was made between this model and RTE, 9 a finite-difference based conjugate heat transfer program. RTE is capable of modeling the two-dimensional steady state temperature profile at up to 61 pre-defined stations of a regeneratively-cooled nozzle, as well as the one-dimensional axial conduction between stations. Model inputs for the comparison included coolant type, coolant inlet pressure and temperature, nozzle geometry, wall material properties, and heat flux from the hot hydrogen. Heat flux profiles were generated in an Excel spreadsheet using a heat transfer coefficient computed from the Bartz equation and based on wall temperatures obtained through iteration with RTE. The iteration was conducted until the maximum wall temperature change at any station between two successive runs was within approximately $0.5^{\circ} \mathrm{F}$.

The primary model outputs of interest were wall temperatures and coolant exit pressure and temperature. All of the results matched very well (Figs. 4a-c). In the figures, a negative value on the $\mathrm{x}$-axis refers to a location upstream of the throat. As shown in Fig. 4a, the calculations for the total pressure drop in the coolant are within $6 \%$, and both pressure curves indicate the same trends. The temperature rise in the coolant is also very close, as seen in Fig. 4b. The hot gas wall temperature is the least accurate parameter (Fig. 4c). The difference is primarily due to the heat transfer in the throat region, which is heavily dependent upon heat transfer enhancement due to curvature effects. Any small differences in this effect can produce large differences in the wall temperature. Even with these differences, the nozzle cooling model's wall temperature is very close to RTE's.

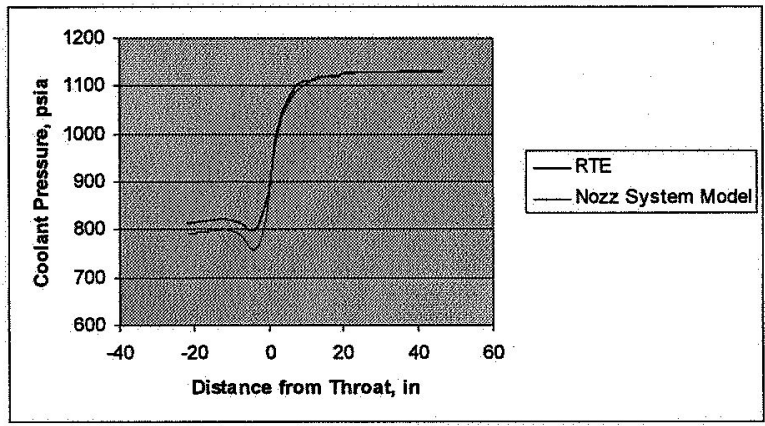

a)
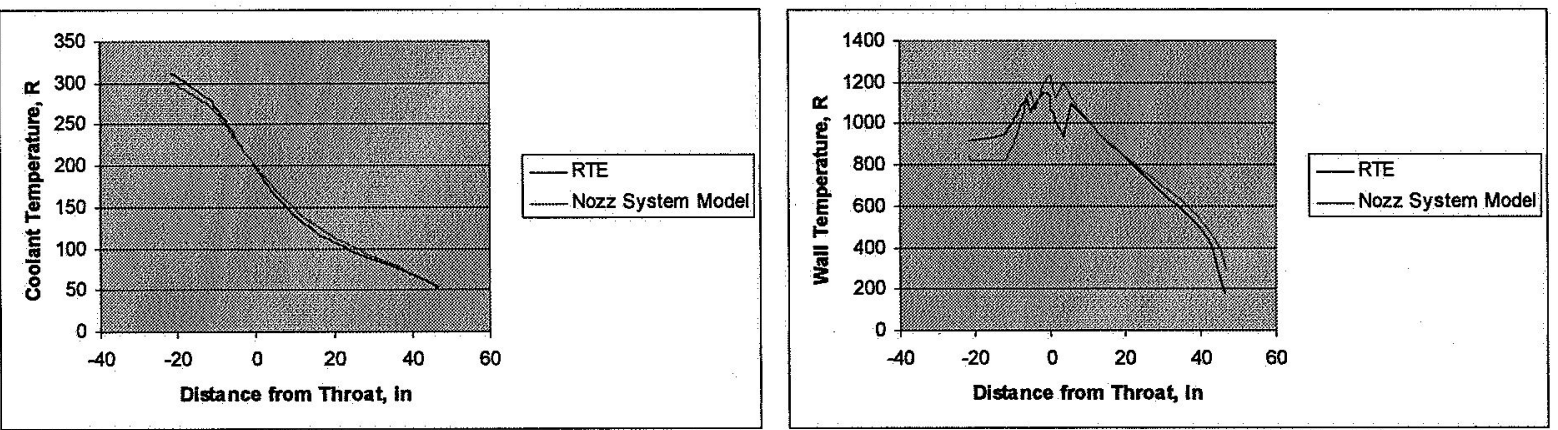

b)

c)

Figure 4. Comparison of Chamber/Nozzle Model in ROCETS to RTE.

\section{New Detailed Reactor Model}

Enabler has some inherent limitations that can affect the design of the reactor. Since the reactor is around $70 \%$ of the total engine weight, these shortcomings can drastically affect the overall performance of the engine. An example of these limitations is Enabler's inability to determine what effect core void fraction, core $L / D$, hex dimension, and moderator amount has on criticality. Another example is Enabler's assumption of a fixed power deposited in each of the reactor's components: core, tie tube/slats, reflector, and shield. Because of these limitations, a new reactor model is being developed to take all of these effects into account. MSFC chose to team with Los Alamos National Laboratory (LANL) to develop this model because of their vast experience with space reactor design.

There are essentially three steps that take place in the new reactor model. The first step is to determine the correct boundary conditions for both the core and tie tubes. The next step is to determine designs that thermally and hydraulically meet the boundary conditions. The last step is to use the Monte Carlo Neutral Particle (MCNP) code to determine a critically acceptable system with the lowest mass. These steps are shown in Fig. 5.

The core's boundary conditions consist of $\Delta P$, exit pressure, inlet temperature, exit temperature, and flow rate. The tie tube and slats' boundary conditions consist of $\Delta P$, inlet pressure, inlet temperature, and flow rate. In order to 
determine the correct limits on the boundary conditions for Step 1 and limit the number of conditions the new reactor model had to analyze, the system model was utilized to determine the maximum core inlet pressure that still could achieve a power balance between the turbine and pump. Different engine cycles (e.g., closed cycle, open cycle, bleed cycle, tie tube flow to the turbine, or nozzle flow to the

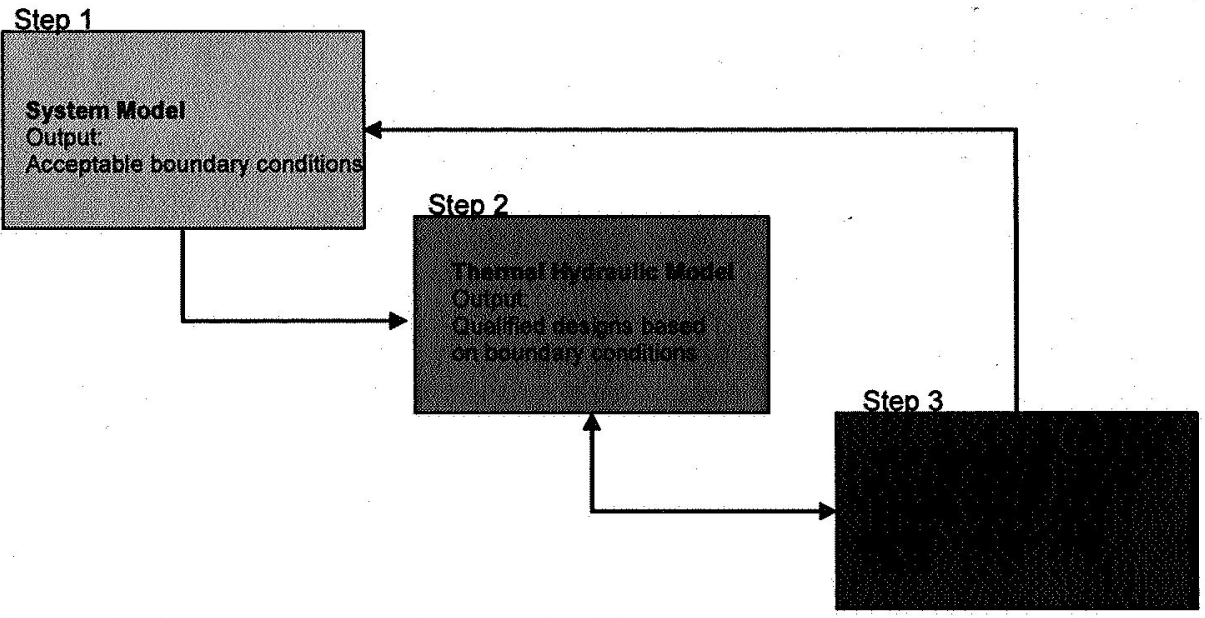

Figure 5. Sequence for New Reactor Model.

turbine) are treated differently. For example, a closed expander cycle was modeled with the turbine being powered by the tie tubes and slats. Since a different maximum core inlet pressure could be obtained for different tie tube and slat powers, a series of tie tube and slat powers had to be run to determine the maximum core inlet pressure. Furthermore, different flow rates through tie tubes and slats could also affect the maximum core inlet pressure, and had to be run. For these runs, a thrust level, core exit temperature, and maximum tie tube and slat $\Delta P$ is assumed. Fig. 6 shows the results of these runs for a $75 \mathrm{klbf}$ thrust engine with tie tube $\Delta P=300 \mathrm{psid}$. The tie tube power is given as a fraction of the total reactor power. From this plot, the amount of flow through the tie tube does not seem to affect the maximum core inlet pressure for this engine cycle. Therefore, only three values for the maximum core inlet pressures and the associated system parameters need to be passed to the next step of the new reactor model.

In Step 2, the thermal hydraulic model takes the boundary conditions from Step 1 and varies the core void fraction, $L / D$ of the core, hex dimension, and number of hexes to find a system that will not exceed the maximum core inlet pressure and satisfies all of the system parameters. In addition, a stress model for the tie tube support is used to make sure the core will be supported no matter what load is placed on it. The output generated by the thermal hydraulic model is shown in Fig. 7. This plot gives acceptable designs for different number of hexes and core fuel volumes. Relations for coolant hole diameter versus core height are also generated in this step.

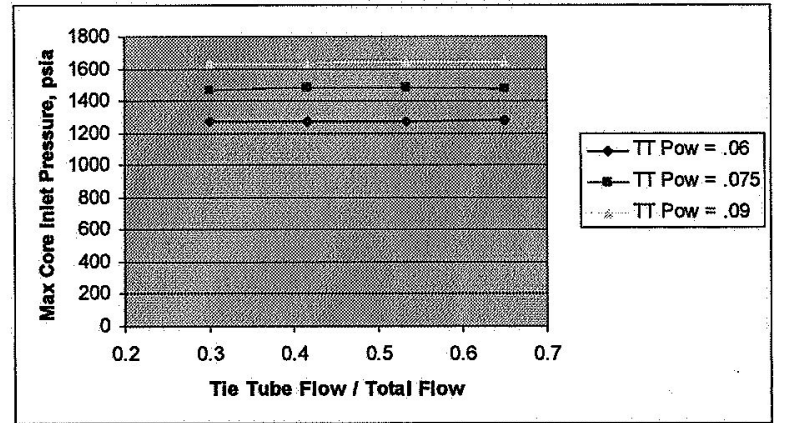

Figure 6. Maximum Allowable Core Inlet Pressure.

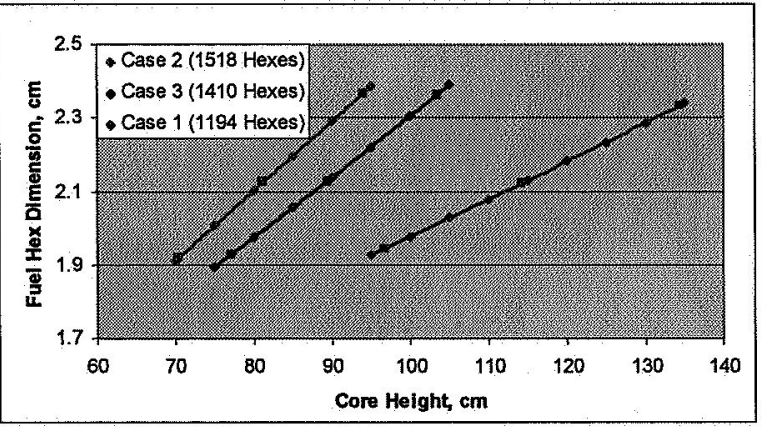

Figure 7. Thermal Hydraulic Model Output.

In Step 3, the output from Step 2 is passed to the nuclear model, which utilizes MCNP. The MCNP tool will use a combination of discrete and partially homogenized models to determine the beginning of life hot $k_{e f f}$ and beginning of life shutdown $k_{e f f}$. An adequate difference between these two values must be obtained. A model of an internal shield on top of the core was inserted into the MCNP tool. The shield can be sized according to flux limits imposed by requirements. Once suitable designs have been found, the lowest mass system will be passed back to the system model so the entire system performance can be ascertained. Fig. 8 shows a portion of the layout of the discrete model in MCNP with enrichment zones that help flatten the radial power distribution.

Through all of these steps, power distributions in the core and the power deposited in the components are assumed. If the MCNP model determines these assumptions are incorrect, an iteration of the whole process must be 
performed. Some preliminary results have already been obtained. Fig. 9 shows the thrust to weight ratio $(T / W)$ of a closed expander cycle engine for a thrust range of 15 to $250 \mathrm{klbf}$. One curve is for the Enabler model while the other curves are for the new reactor model with two different weight percent loadings of uranium. This plot shows a large increase in $T / W$ using the new, more accurate model. The main reason for this increase is due to the fact that more parameters (e.g., coolant hole diameter, hex dimension, fuel loading, etc.) can be varied to obtain an improved design.

Unfortunately, with a detailed model like MCNP in the process, it could take hours to arrive at a solution, even if portions of the reactor are homogenized. This technique is not practical for performing system trades or responding to quick turn-around design studies, nor was it the original intent for the integrated, fast, reactor model to use MCNP. Initially, the integration of a much faster homogenized deterministic model into the system tool was anticipated. However, inconsistent biasing between the discrete and deterministic models for this type of system forced the analysts to change directions.

As a result, another approach was conceived to develop an integrated, fast, reactor model. This fast model will utilize the results from the detailed model described above. Once the detailed model with MCNP has been exercised to the point where the results for several cases have been validated, a matrix of additional reactor cases will be analyzed. After this database of reactor solutions has been generated, a surface response model will be incorporated so that the system tool can arrive at engine/reactor solutions very quickly. Additional detailed MCNP modeling can continue concurrently for other cases of interest. These new results can be used to refine or fill in additional regions of the database. The additional data will improve the

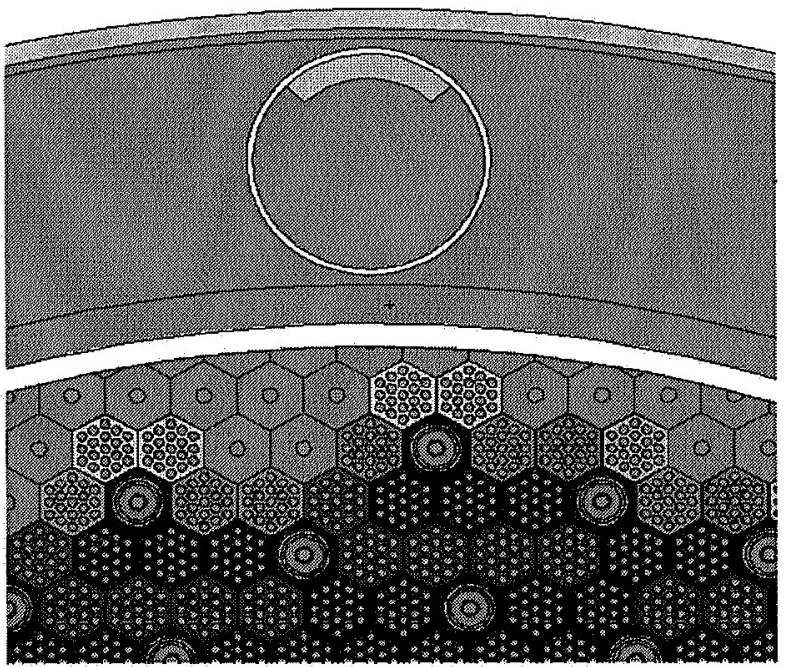

Figure 8. Preliminary MCNP Results. accuracy and flexibility of the surface response model.

After the NERVA-Derived reactor model and database are established, a similar model to design reactors with CERMET prismatic fuel elements will be created. Additional reactor concepts will be modeled over time based on direction from the project office.

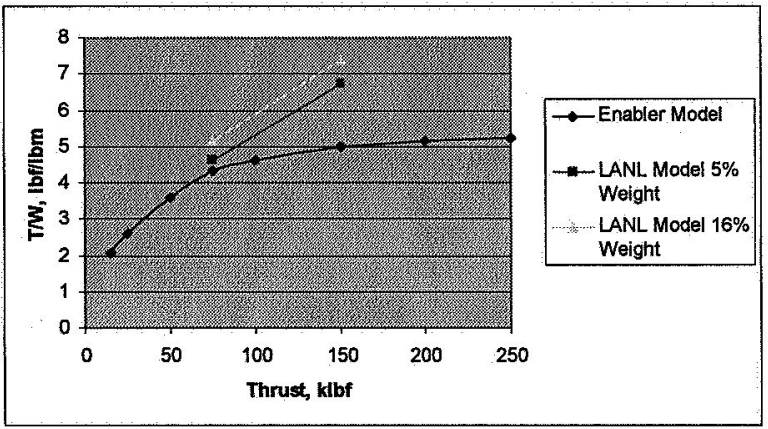

Figure 9. Thrust to Weight Comparison.

\section{Sample Results}

\section{A. Overview}

Approximately 70 NERVA-Derived engine cases have been generated to date based on the trade space that was established for the task. The trade space included engine cycle, thrust, $P c$, and $E R$. Four engine cycles, closed expander with tie tubes feeding the turbine (CX-T0), closed expander with nozzle and reflector feeding the turbine (CX-R0), cold bleed with tie tubes feeding the turbine (CB-T0), cold bleed with nozzle and reflector feeding the turbine (CB-R0), were analyzed. The eight thrust levels ranged from 15 to $250 \mathrm{klbf}$. The baseline $P c$ and $E R$ were 450 psia and 100:1, respectively. Sensitivities were performed on these parameters with $P c$ up to 1000 psia and $E R$ up to $200: 1$.

Each engine case had a reactor core with 0.75 inch flat to flat composite graphite prismatic fuel elements and tie tube support elements. The core length was 52 inches, and the core exit temperature was $2700 \mathrm{~K}$. The fuel element to tie tube ratio was $6: 1$ for engines with $75 \mathrm{klbf}$ thrust and higher. The 15,25 , and $50 \mathrm{klbf}$ thrust engines had an element ratio of $2: 1,2: 1$, and 3:1, respectively. One special CX-T0 case with a thrust of $16 \mathrm{klbf}$, a $P \mathcal{c}$ of $450 \mathrm{psia}$, an $E R$ of 100:1, a core length of 35 inches, and an element ratio of 2:1, was ran to compare the model to the LANL Small Engine design. ${ }^{16}$ 
B. Data Included in Spreadsheets

As mentioned

previously,

comprehensive

spreadsheet is generated

for each engine

concept, or case, which

has a given set of

design characteristics: engine cycle, thrust, $P c$, and $E R$. Fig. 10 is an example of a summary page worksheet from one case that was analyzed and is considered the point of departure (POD). The POD case is a $75 \mathrm{klbf}$ thrust CX-T0 with $P c=$ $450 \mathrm{psia}$, and $E R=$ 100:1. The spreadsheet for each case includes additional worksheets with data, plots, and figures for the following subsystems: reactor, external shield, chamber and nozzle, pump and turbine, and valves and lines.

Also included in the spreadsheet is a drawing page where a scaled and dimensioned cutaway of the engine is generated with the Visual Basic software in Microsoft Excel. The sizes of the reactor components are based on the weight calculation logic that is performed in Enabler. The drawing is intended for qualitative comparisons only. The engine drawing for the POD case is shown in Fig. 11.

A weights page is also included with each spreadsheet that provides a breakdown of the system weight by component. Additional parametrics on weight are performed on this page where various shielding and turbopump configurations are considered. Fig. 12 shows the weight breakdown by component with mass (in $\mathrm{lbm}$ ) and percentage for the POD case with two turbopumps and no external shield.

\section{Performance, Sizing, and} Sensitivities

Figs. 13a-c summarize the results for the baseline cases $\left(P_{c}=450 \mathrm{psia}\right.$, and $E R$ $=100: 1)$. In Fig. 13a, the specific impulse

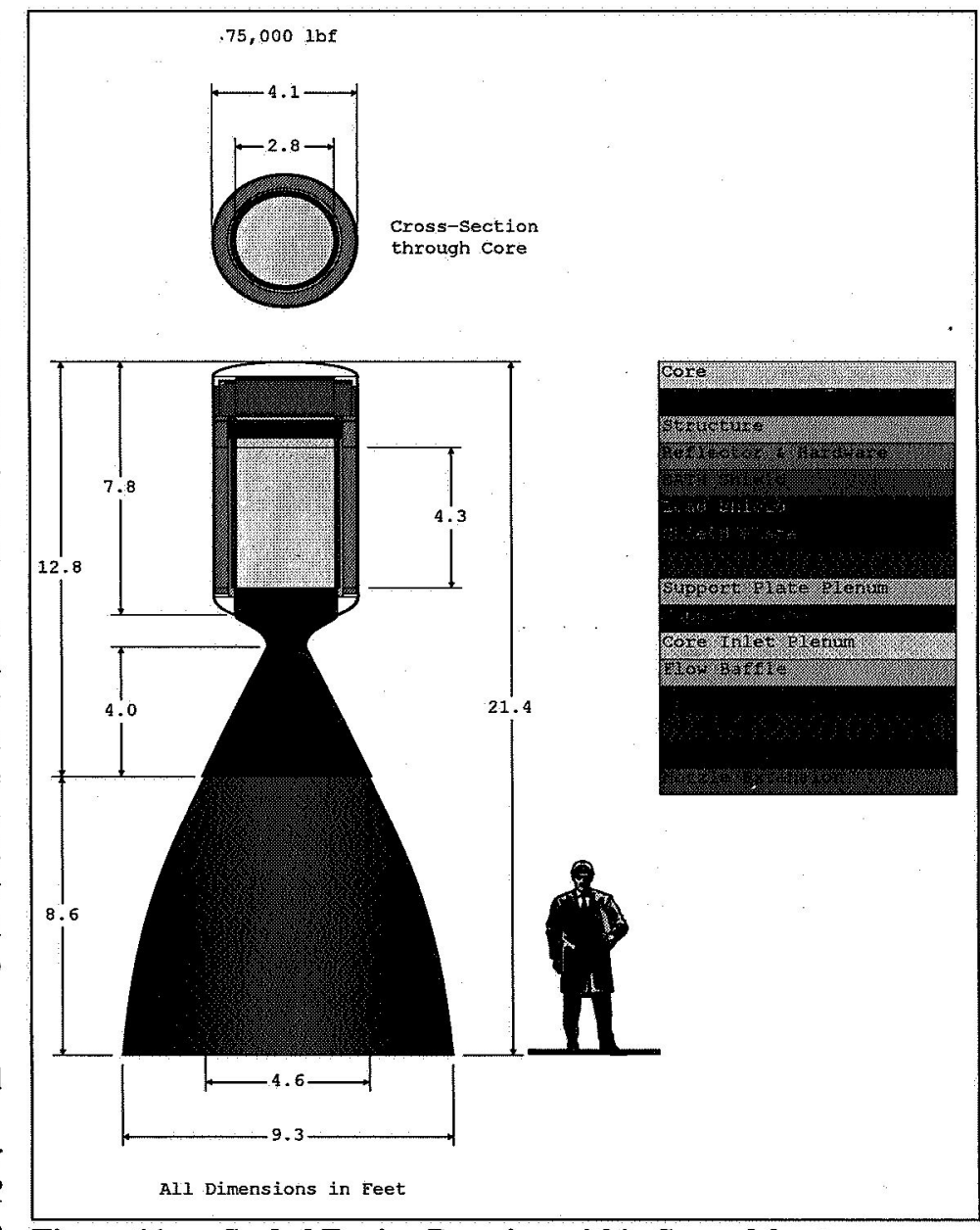

Figure 11. Scaled Engine Drawing within Spreadsheet. 
(Isp) for the closed expander cycles is a respectable 887 seconds. The bleed cycles are lower by approximately $2 \%$. The turbine exhaust for the bleed cycles is injected into the nozzle at the regeneratively cooled section and radiatively cooled section interface where $E R=25: 1$. Notice that thrust levels greater than $100 \mathrm{klbf}$ were unobtainable for CX-R0 due to the lack of power available from the nozzle and reflector coolant flow to drive the turbine.

In Fig. 13b, the $T / W$ for the larger engines was around $5 \mathrm{lbf} / \mathrm{lbm}$, and as low as $2 \mathrm{lbf} / \mathrm{lbm}$ for the $15 \mathrm{klbf}$ thrust engine. Engine weight did not vary significantly with engine cycle.

In Fig. 13c, the nozzle exit diameter was used as a measure of envelope, and is proportional to the square root of the thrust. When clustering engines, this

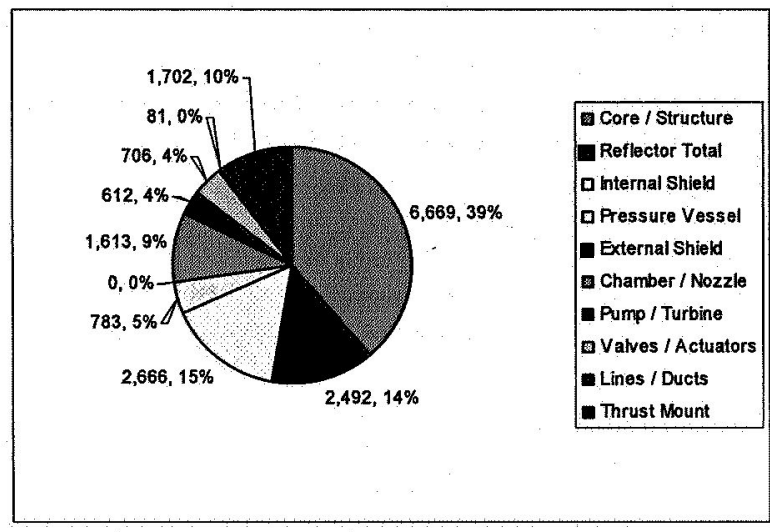

Figure 12. Weight Breakdown by Component. dimension would be the minimum distance from engine axial centerline to centerline. Thus, nozzle diameter is an important factor in the size and weight of shadow shields.

Fig. 13d illustrates the contribution of the various components to the total engine weight. Only the data for the CX-T0 cycles are shown in this figure. The core and its structure, internal shield, reflector, thrust mount, and chamber/nozzle are the primary contributors. Since the reflector thickness is essentially fixed for all thrust levels, its weight contribution increases significantly as thrust decreases. The thrust mount calculation also indicates increasing contribution with decreasing thrust. The core and structural mass contribution tends to decrease at the low thrust range, but varies due to the changes in the element ratio. The chamber/nozzle contribution decreases dramatically with decreasing thrust. For all thrust levels, the reactor components contribute to roughly $70 \%$ of the total engine weight.
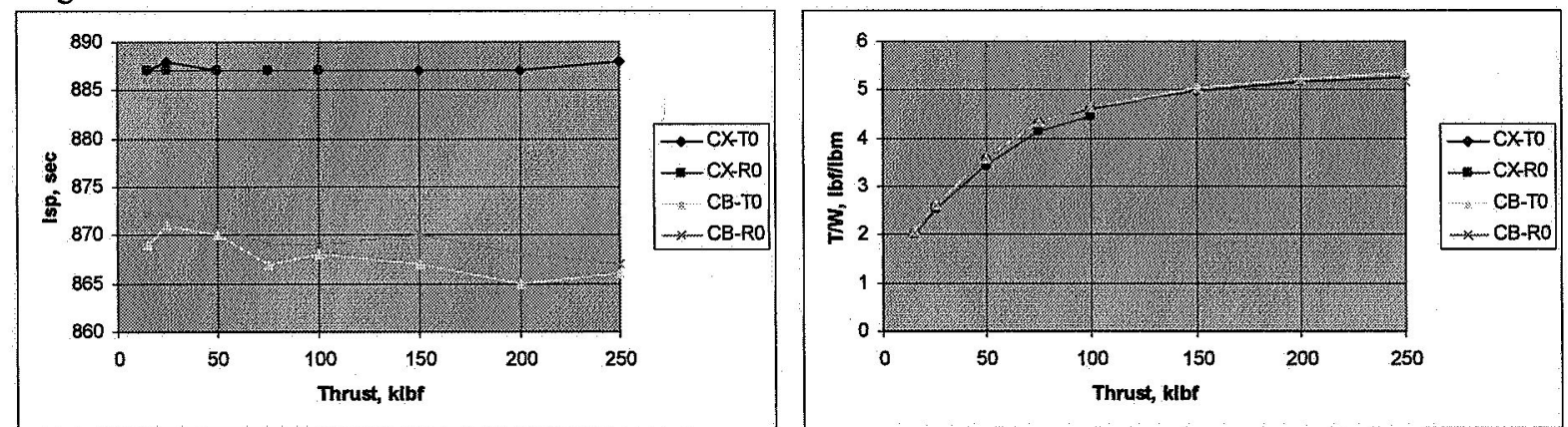

a)

b)
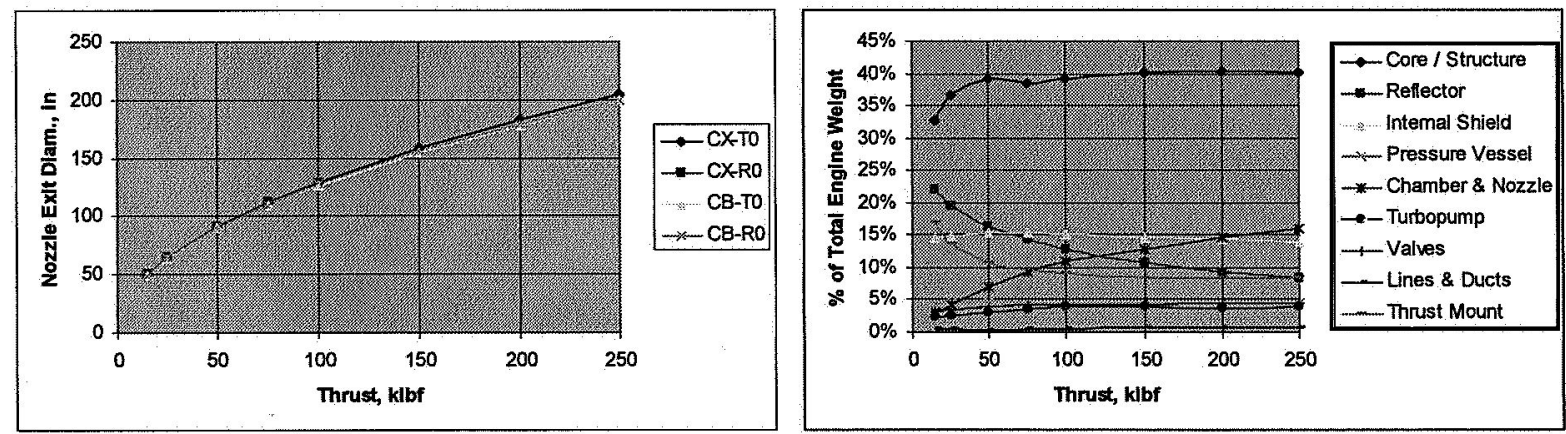

c)

d)

Figure 13. Performance Results at Various Thrust Levels.

Sensitivities on engine performance were analyzed by varying $P c$ and $E R$ at select thrust levels. Figs. 14a-d show the effects these parameters have on $T / W, I s p$, and envelope for a $75 \mathrm{klbf}$ thrust CX-T0 engine. As expected, 
increasing $E R$ improves $I s p$, and $I s p$ is independent of $P c$, as shown in Fig. 14a. From Fig. 14b, $T / W$ peaks at a $P c$ of around $750 \mathrm{psia}$, and $T / W$ is only slightly affected by $E R$. Although the modeling does show some impact on performance as a result of varying these parameters, the performance increases are small in a relative sense. The $I_{s p}$ increase due to the $E R$ increase from $100: 1$ to $200: 1$ is only $1 \%$. The $T / W$ increase due to the $P c$ increase from 450 to 750 psia is also only $1 \%$. Plus, the effects on the system of increasing $P c$ may actually reduce the engine reliability.

Increases in $E R$ and $P c$ have conflicting impacts on envelope. As indicated by Fig. 14c, the nozzle exit diameter increase due to the $E R$ increase from 100:1 to $200: 1$ is $41 \%$. As indicated by Fig. 14d, the nozzle exit diameter decrease due to the $P c$ increase from 450 to $1000 \mathrm{psia}$ is $33 \%$. Obviously, the entire engine system, mission, vehicle, and shielding approach need to be considered when selecting the appropriate engine design requirements.

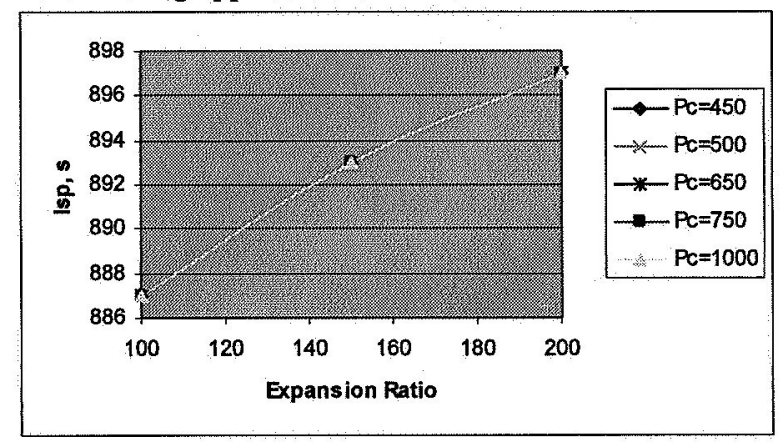

a)

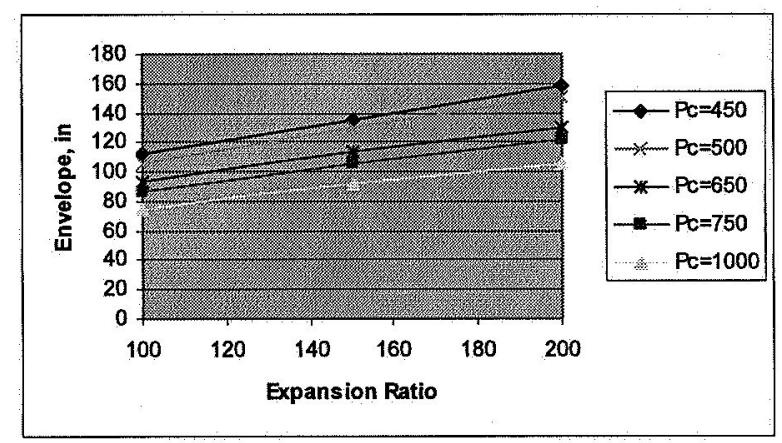

c)

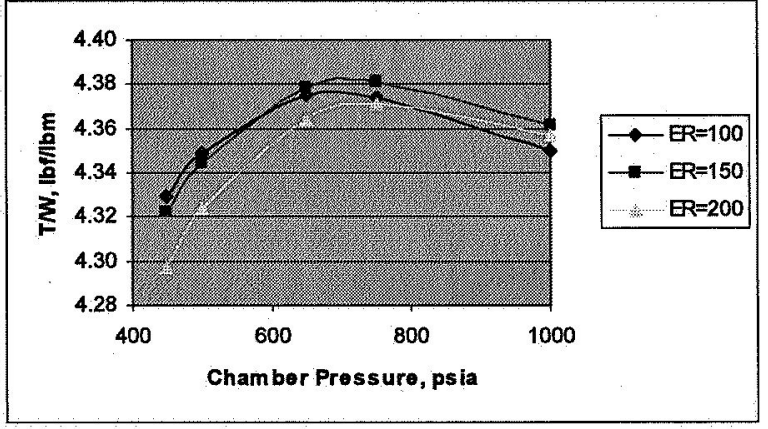

b)

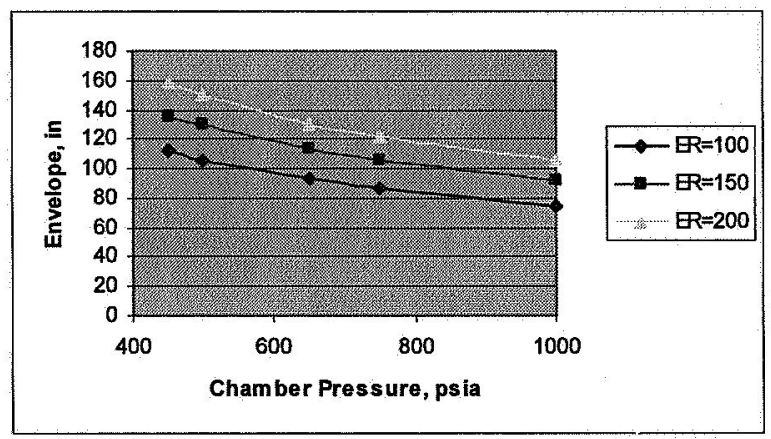

d)

Figure 14. Sensitivity Results for 75 klbf Thrust CX-T0.

\section{Conclusion}

Designing an engine system for an interplanetary mission, especially when manned, is very complex with numerous variables and interactions. All FOMs (performance, reliability, cost, safety, and risk) must be considered in the engine system design. In addition, engine impacts on the overall vehicle and trajectory must be assessed through trades and sensitivities. The newly implemented NTP design and analysis tool is very powerful and will aid in the development and direction of NTP. Improvements, which are currently underway, such as the detailed reactor model and system reliability model, will even further help with future design decisions and requirements definition. Once the reactor reliability model is complete, the baseline cases and sensitivities that have been run to date will be compared based on performance, reliability, and, to a limited extent, risk and cost. To perform this initial evaluation, an engine FOM process will be developed to determine the appropriate weighting of the parameters. A more detailed cost model, a task that was de-scoped from the project due to funding limitations, will be added to the tool sometime in the future.

The parameter with the most influence on engine performance is the core exit temperature. Increasing temperature will increase Isp and reduce IMLEO. More advanced core designs and fuel materials (e.g., CERMET, carbide, foam core, etc.) are being investigated to do just that and reduce weight as well. These concepts will soon be modeled with an improved version of the current tool. In the meantime, the $T / W$ performance of the NERVADerived systems can be addressed by exploring ways to decrease system weight, particularly in the reactor. The improved reactor model will provide more accurate reactor weights and a better understanding of the key design 
parameters that influence the weight. Preliminary results with the newly implemented model show that a reactor designed for a specific power level will weigh less than what current reactor models are predicting. This difference is due to the fact that current models are anchored to only reactors that were previously designed, built, and tested, and are not capable of simulating more optimized reactor geometries.

A 3-D CAD model of the NERVA-Derived $75 \mathrm{klbf}$ thrust POD engine is currently being developed to gain an understanding of the detailed design, external plumbing layout, and final assembly. The model will later be parameterized so that engines at other thrust levels and with other reactor geometries can be generated. The model will also be used to verify component and subsystem weights obtained from the design tool.

The shielding approach for these manned missions continues to be a very complex problem. In addition to radiation from the engine, shielding studies need to account for galactic cosmic radiation (GCR) and shielding already required on the crew module for GCR. If a large external engine shield is required to achieve acceptable radiation exposure limits, the lower engine $T / W$ will greatly affect the vehicle and mission, and increase program cost and risk. Clustering multiple small engines rather than using one larger engine results in lower $T / W$. External shielding for clustered engines amplifies this effect. Preliminary studies performed for the NTP engine system task have shown that a single engine/reactor with two turbopumps is more reliable than a multiple engine configuration. In addition, since the reactor makes up the majority of the engine mass, the weight of the additional pump has little effect on $T / W$. However, performance, engine development cost, reliability, vehicle/trajectory impacts, and overall program cost need to be examined further to determine if engine clustering is beneficial.

\section{Acknowledgments}

Initial financial support for this effort was received from the MSFC Independent Research and Development (IR\&D) Program in March 2005, with additional IR\&D support in 2006. The MSFC Nuclear Systems Office (NSO) selected this task for additional funding from the Prometheus Nuclear Systems and Technology Office beginning in June 2005. The NSO/Prometheus support has allowed additional scope and modeling detail, and an integrated effort among other MSFC NTP tasks.

The authors would like to thank the other members of the NTP engine system task team and participants from industry and academia: Mr. Brad Bullard, Mr. Shawn Fears, Mr. Ray Moye, and Mr. Danny Weldon from MSFC; Mr. Rick Kapernick, Mr. Tom Marcille, Mr. Pratap Sadasivan, and Mr. Ben Amiri from LANL; Dr. Robert Chiroux from SAIC; Dr. Samim Anghaie from the University of Florida; Mr. Mike Owens from ERC; and Ms. Katherine Yakubisin, NASA Academy Research Associate from the University of Virginia.

Finally, the team would like to thank Mr. Wayne Bordelon, MSFC NTP Project Manager, for his excellent leadership, knowledge, and enthusiasm.

\section{References}

${ }^{1}$ Leahy, J., "Features and Results Using P-STAR: A Propulsion Sizing, Thermal, Accountability and Weight Relationship First Order Modeling Tool," 52nd JANNAF Propulsion Meeting, May 2004.

${ }^{2}$ Pelaccio, D. G., Scheil, C. M., and Petrosky, L. J., "Nuclear Engine System Simulation (NESS): Version 2.0, Program User's Guide," NASA Contractor Report 191081, NASA Contract No. NAS3-25809, Mar. 1993.

3"'ROCETS User's Manual," Pratt and Whitney, West Palm Beach, FL, Jan. 1995.

${ }^{4}$ Koenig, D. R., "Experience Gained from the Space Nuclear Rocket Program (Rover)," Los Alamos National Laboratory report LA-10062-H, May 1986.

${ }^{5}$ Wolf, H., McCarthy, J. R., "Heat Transfer to Hydrogen and Helium with Wall to Fluid Temperature Ratios to 11.09," Research and Development Program on Thixotropic Propellants, Quarterly Progress Report RP-60-12, Aeroprojects, Inc., West Chester, PA, 1960.

${ }^{6}$ Haaland, S. E., "Simple and Explicit Formulas for the Friction Factor in Turbulent Pipe Flow," J. Fluids Eng., Mar. 1983, pp. 89-90.

${ }^{7}$ Glassman, A. J., "Computer Program for Preliminary Design Analysis of Axial-Flow Turbines," NASA-TN-D-6702; Mar. 1972.

${ }^{8}$ Glassman, A., "Computer Code for Preliminary Sizing Analysis of Axial-flow Turbines," NASA CR-4430, 1992.

'Naraghi, M. H. N., "User Manual for RTE2004 Version 1 - A Computer Code for Three-Dimensional Rocket Thermal Evaluation."

${ }^{10}$ Bartz, D. R., "Turbulent Boundary-Layer Heat Transfer from Rapidly Accelerating Flow of Rocket Combustion Gases and of Heated Air," Advances in Heat Transfer, 1965, pp. 2-108.

${ }^{11}$ Eckert, E. R. G., and Drake, R. M., Analysis of Heat and Mass Transfer, McGraw-Hill Book Company, 1972.

${ }^{12}$ Chen, N. H., "An Explicit Equation for Friction Factor in Pipe," Ind. Eng. Chem. Fundam., Vol. 18, No. 3, 1979, pp. 296297. 
${ }^{13}$ Huzel, D. K., and Huang, D. H., Modern Engineering for Design of Liquid-Propellant Rocket Engines, AIAA, Washington, DC, 1992, p. 76.

${ }^{14}$ Chiroux, R. C., "Reactor Shield Subroutine XTSHIELD User's Manual \& Programming Guide," Science Applications International Corporation, Huntsville, AL, Dec. 2005.

${ }^{15}$ Moyers, J. C., Nichols, J. P., "ALKASYS, A Computer Program for Studies of Rankine-Cycle Space Nuclear Power Systems," ORNL/TM-10427, Sep. 1987.

${ }^{16}$ Durham, F. P., "Nuclear Engine Definition Study Preliminary Report," Volumes I - III, Los Alamos Scientific Laboratory report LA-5044-MS, Sep. 1972. 